\title{
RELATIVE TOLERANCE OF EGYPTIAN CLOVER GENOTYPES TO DODDER INFESTATION
}

EI-Refaey, R.A. ${ }^{1}$; E. H.El-Seidy ${ }^{1}$; I.ESoliman ${ }^{2}$ and Shereen M. EI-Nahrawy ${ }^{3}$

Agron. Dept., Fac. of Agric., Tanta Univ., Egypt.

Weed Res. Central Lab., Agric.Res.Cent , Giza, Egypt.

Forage Crops Res. Dept., Field Crops Res. Inst., ARC, Egypt.

\begin{abstract}
Egyptian clover or berseem (Trifolium alexandrinum L.) is the principle forage crop in Egypt. Unfortunately, the uncertified and uncontrolled local seeds are being contaminated with dodder (Cuscuta spp.) seeds, which affecting negatively both productivity and quality of produced forage. Three field experiments were carried out at Sakha Agricultural Research Station Farm, Kafer El-Sheikh Governorate, ARC, Egypt, during three successive winter seasons; 2008/09, 2009/10 and 2010/11, to investigate the performance of 100 berseem genotypes; 96 farmers seed lots and four commercial cultivars for its resistance/tolerance to dodder infestation. Highly significant differences were observed among the 100 genotypes under evaluation as well as the interaction between the sets and genotypes for all cuts and seasonal yield in the $1^{\text {st }}$ season. The commercial cultivar; Helali (Genotype no.111) has high potential and high-yielding ability in comparison with other cultivars and landraces. It was bred for relatively high ability to rapid re-growth which may be associated with tolerance to biotic and abiotic stresses. In addition to, some landraces retained the least reduction percent of total chlorophyll. In general, the berseem genotypes under investigation manifested highly significant differences at all cuts and seasonal fresh and dry yield as well as chlorophyll content for reduction percent due to dodder infestation.
\end{abstract}

\section{INTRODUCTION}

Egyptian clover or berseem (Trifolium alexandrinum L.) is a winter annual legume widely grown in several countries for fresh fodder, hay and silage. In Egypt, the most limiting factor for animal wealth development is the lack of local feed due to the wide gap between the consumed and available local feed sources (ACSAD, 2008). Therefore, berseem is not only endogenous to Egypt but also the principal forage crop and occupying about 2.0-2.8 million Fadden (El-Nahrawy, 2009b). The animal wealth development is depending mainly on berseem in the winter season because most of the animal protein requirement is fulfilled with feeding on berseem (ACSAD, 2008). It is considered a principal source of animal feed for the most whole year specifically the smallholders due to its high yielding and quality potential. Moreover, it is the fertility foundation of agriculture in the Nile Delta and the valley and playing a vital role in sustaining the Egyptian agriculture (ElNahrawy, 2009a). In addition, berseem still considered indispensable in rotation with cotton and other crops due to its high nitrogen fixation potential, so, it fixes more than 714 thousand tons of atmospheric N2 annually in Egypt (Kennedy \& Mackie, 1925; Abd EL-Hady, 1993; Graves et al, 1996). Furthermore, berseem is one of the important sources for honey bees foraging. Nevertheless, berseem has not received much attention compared to cereal crops; i.e. wheat, rice and corn (The Strategy for Sustainable 
Agricultural Development Towards; 2030). The remarkable increase in cereal productivity in the last two decades (from 8 million MT in 1980 to more than 22 million MT in 2009) is mainly due to developing high-yielding cultivars and making their certified seed available to growers. Unfortunately, this is not the case for berseem. Farmers have traditionally produced their own seed or purchased their requirements from the local markets. However, local seed is both uncertified and uncontrolled and the quality of such seed on local market is rather poor and most of it is contaminated with many weed seeds, especially, dodder seeds (Cuscuta spp.). Recently, an increasing number of farmers $(85 \%)$ have been reporting troubles due to dodder infestation in berseem fields which affect both productivity and quality of produced forage (Abd El-Hamid \& El-Khanagry, 2006). Dodder affects the growth and yield of infested plants and causes losses in crop. Infested berseem with dodder could lead to reduced protein content, fresh as well as dry forage yield and nutritive value. Therefore, dodder is considered a very big problem overall the world including Egypt and one of the main parasitic weed for crop plants not only berseem but also alfalfa, vegetables and fruits. Dodder can germinate alone if the weather is fit to germination, but, it cannot survive alone without attachment to a host. It must attach itself with a host plant by the suckers of the dodder. The hard seeds of dodder can remain in the soil for many years viable (Abd El-Wahed, 1996). Dodder species are distributed worldwide and continued to attack many different host plants. International trade, mainly with contaminated crop seeds, has led to the wide distribution of this parasite (Parker \& Riches, 1993). It lives entirely on the host plant thus reducing the growth and yield of the host. Several herbicides have been successfully shown to selectively suppress attached dodder but complete control is rarely obtained. Dodder attached to genetically modified, herbicide resistant crops; have not been successfully killed by treatment with herbicide in all cases, indicating that, these crops will only be a partial solution to the problem. Dodder control will require an integrated approach conducted over a period of many years (Lanini \& Kogan, 2005). Therefore, the main goals of this study were to assess the effect of dodder on growth and development of available berseem germplasm; commercial and high-yielding developed cultivars as well as farmers' seed lots. In addition to, estimate the losses occurred due to dodder infestation and to identify the most tolerant genotypes under artificial infestation.

\section{MATERIALS AND METHODS}

Three field experiments were carried out at Sakha Agricultural Research Station Farm, Kafer El-Sheikh-Governorate, Forage Crops Research Department (FCRD), Field Crops Research Institute (FCRI), Agricultural Research Center (ARC), Egypt, during three successive winter seasons; 2008/09, 2009/10 and 2010/11 to investigate the performance of some Egyptian clover genotypes for forage yield and its tolerance to dodder infestation (Cuscuta planiflora). One hundred genotypes were used in this investigation. Ninety six genotypes were chosen randomly from big collection of berseem farmers' seed lots (landraces) through survey overall most of the 
country issued by FCRD to capture most of the existing genetic variation and the rest four genotypes were commercial cultivars; Helali, Sakha-4, Sids-1 and Serw-1.

In the $1^{\text {st }}$ season (2008/2009): Simultaneously, two adjacent as well as similar layouts were conducted. The recommended cultural practices for berseem such as preparing good seed bed, adding NPK fertilizers, cutting and irrigation were applied. The first layout accommodated the 100 genotypes which were divided into five sets and each set contained 20 genotypes. Genotypes were randomly distributed within each set and sets were randomly distributed within each replicate of the four replicates in complete block design without infestation of dodder seed. The second layout was carried out in a similar way to the first one but berseem seeds of each plot were mixed with dodder seeds in rate of $5 \%$ of berseem seeds, as it stated by Soliman (2002) to achieve the artificial infestation. The plot size was $1.2 \times 2.0 \mathrm{~m}$ which contained four rows. The seeds of each genotype were seeded within the four rows according to the layout of the experiment. The space between the rows was $30 \mathrm{~cm}$ and $1.50 \mathrm{~m}$ between the plots. The sowing dates of the experiments were October 20, 15 and 18 for the three seasons, respectively. Four cuts were taken. Fresh forage yield/plot were weighted (Kg/plot) in the non-infested experiment and after separating dodder material from forage in the infested experiment. Percentage of reduction $(R$ $\%$ ) in forage yield due to dodder infestation was calculated according to Topps \& Wain (1957) formula as following: $R \%=(A-B / A) \times 100$.

Where; A: Fresh forage weight on non-infested,

$B$ : Fresh forage weight on infested.

At the end of $1^{\text {st }}$ season, 25 genotypes were selected based on the least value of reduction in descending order of the fresh forage yield out of the 100 genotypes under evaluation. After the $4^{\text {th }}$ cut, the selected 25 genotypes were left for open cross-pollination and the rest of genotypes were discarded to eliminate the possibility of producing pollen and providing good isolated area for the selected genotypes. Three hives of honey bees were provided during the flowering time to insure intercross pollination among the selected genotypes. Seeds of each genotype of the 25 selected genotypes were harvested, separately, after reaching maturity stage.

In the $2^{\text {nd }}$ season (2009/2010): Seeds of the selected 25 genotypes along with two lots; genotypes no. 29 and 94, which were identified to be very sensitive to dodder infestation in the $1^{\text {st }}$ season evaluated in a randomized complete block design (RCDB) with four replicates. The plot size, number of rows within the plot, the distance between plots and number of cuts were similar to that in the $1^{\text {st }}$ season. At the end of $2^{\text {nd }}$ season, selection was conducted for the genotypes that had the least percent of reduction in descending order. Six genotypes were selected based on the least value of reduction of the fresh and dry forage yield out of the 25 genotypes under evaluation. Seeds of each genotype of the six selected genotypes were harvested, separately. Reduction percentages for fresh and dry forage yield were estimated. Total chlorophyll content was determined from 10 fresh berseem plants mechanically by using chlorophyll meter content (spade 
value) for 10 genotypes which represent four degrees of dodder infestation tolerance; high, medium, low tolerance and sensitive. Also, percentage of reduction for total chlorophyll was estimated.

In the $3^{\text {rd }}$ season (2010/2011): remnant seeds of the selected six genotypes which represent the parents as well as seeds of the same genotypes which selected in the $2^{\text {nd }}$ season which represent the progenies of these parents and two check commercial varieties Giza-6 and Gemmiza-1 were evaluated on a RCDB with four replicates as stated in the $2^{\text {nd }}$ season. The materials were left for seed production in isolated area and few honey bees' hives were provided during flowering time. Percentages of reduction for fresh as well as dry forage yield were estimated.

Analysis of variance for RCDB with four replications was carried out according to Sendecor \& Cochran (1971) for each experiment (infested and non-infested). Moreover, the variance components were calculated according to Comstock \& Robinson (1952).

\section{RESULTS AND DISCUSSION}

\section{$1^{\text {st }}$ season (2008/2009)}

Highly significant differences among the 100 genotypes under evaluation, the interaction between the sets and genotypes for all cuts and seasonal yield had been detected (Table 1). Means of fresh forage yield reduction percentages (FFY R \%) due to infestation with dodder for the 100 genotypes at the four cuts and seasonal yield in the $1^{\text {st }}$ season $(2008 / 09)$ are shown (Table 2). FFY R \% ranged from 4.75 for genotype no. 111 to about 54.0 for genotype no.108 at the $1^{\text {st }}$ cut. Moreover, FFY R \% ranged from 10.2 for genotype no.51 to 54.6 for genotype no.32 at the $2^{\text {nd }}$ cut. In addition, FFY $\mathrm{R} \%$ ranged from 8.93 for genotype no. 51 to about 46.35 for genotype no.70 at the $3^{\text {rd }}$ cut. While, FFY R\% ranged from 14.03 for genotype no. 51 to 61.9 for genotype no.13 in the $4^{\text {th }}$ cut. Regarding seasonal forage yield, R\% value ranged from 11.44 for genotype no.51 to 47.9 for genotype no.32. In general, genotype no.51 has the least reduction value $(R \%)$ among all the genotypes at all cuts and seasonal yield except at $1^{\text {st }}$ cut, where, it is preceded by genotype no.111 which gave $4.75 \%$ for FFY then followed by genotype no.74, where, it gave $5.26 \%$. Moreover, the average of FFY R \% across the cuts increased in ascending order i.e. $\mathrm{R} \%$ at $4^{\text {th }}$ cut $>3^{\text {rd }}$ cut $>2^{\text {nd }}$ cut $>1^{\text {st }}$ cut. However, the highest FFY R \% value was manifested by genotype no. 13 , where, it gave 61.9 at $4^{\text {th }}$ cut and genotype no. 94 where it gave 50.56 regarding the seasonal yield.

Table 1 : Mean squares of FFY R \% for Egyptian clover genotypes infested by dodder at different cuts and seasonal yield in $1^{\text {st }}$ season (2008/09).

\begin{tabular}{|c|c|c|c|c|c|c|}
\hline \multirow{2}{*}{ S. o.v } & \multirow{2}{*}{ d. f. } & \multicolumn{5}{|c|}{ Mean squares } \\
\cline { 3 - 7 } & & Cut1 & Cut2 & Cut3 & Cut4 & Seasonal yield \\
\hline Sets (S) & 4 & 104.118 & 333.257 & 847.456 & 1309.798 & 341.716 \\
\hline Reps/S & 7 & 120.271 & 353.056 & 868.245 & 1331.266 & 353.217 \\
\hline Genotypes(G) & 19 & $961.623^{\prime \prime}$ & $388.440^{\prime \prime}$ & $292.503^{\prime \prime}$ & 864.297 & 411.520 \\
\hline S x G & 76 & $538.991^{\prime \prime}$ & 274.575 & 252.966 & 461.00 & 283.900 \\
\hline Error & 258 & 8.485 & 10.870 & 10.877 & 3.920 & 4.768 \\
\hline
\end{tabular}

${ }^{* *}$ : Significant at the 0.01 level of probability. 
Table 2 : Means of FFY R \% of Egyptian clover genotypes infested by dodder at different cuts and seasonal yield in $1^{\text {st }}$ season (2008/2009).

\begin{tabular}{|c|c|c|c|c|c|}
\hline Genotype & $1^{\text {st }}$ Cut & $2^{\text {nd }}$ Cut & $3^{\text {rd }} \mathrm{Cu} 3$ & $4^{\text {th }}$ Cut & Seasonal yield \\
\hline 1 & 44.725 & 34.900 & 32.675 & 44.425 & 38.328 \\
\hline 2 & 33.00 & 26.375 & 13.475 & 28.750 & 24.745 \\
\hline 4 & 37.700 & 37.625 & 32.150 & 41.975 & 37.238 \\
\hline 6 & 43.625 & 40.100 & 33.450 & 30.450 & 36.338 \\
\hline 7 & 48.325 & 45.750 & 39.275 & 49.725 & 27.477 \\
\hline 8 & 27.425 & 24.900 & 23.875 & 33.725 & 27.477 \\
\hline 9 & 44.625 & 36.700 & 34.325 & 52.500 & 42.198 \\
\hline 10 & 47.525 & 46,625 & 43.350 & 50.075 & 46.725 \\
\hline 11 & 35.975 & 39.775 & 36.275 & 59.850 & 43.840 \\
\hline 12 & 16.750 & 14.925 & 16.625 & 27.525 & 19.160 \\
\hline 13 & 40.025 & 40.625 & 28.100 & 61.900 & 42.945 \\
\hline 14 & 8.103 & 29.900 & 18.100 & 18.950 & 20.253 \\
\hline 15 & 15.100 & 19.100 & 15.875 & 14.875 & 16.395 \\
\hline 16 & 6.450 & 27.400 & 21.625 & 17.975 & 20.055 \\
\hline 17 & 39.200 & 29.900 & 26.45 & 43.250 & 34.047 \\
\hline 18 & 26.575 & 33.325 & 26.975 & 32.125 & 30.563 \\
\hline 19 & 34.450 & 41.125 & 35.800 & 32.975 & 35.477 \\
\hline 20 & 29.225 & 28.775 & 30.350 & 27.200 & 28.920 \\
\hline 21 & 9.200 & 30.975 & 16.275 & 36.325 & 25,023 \\
\hline 22 & 24.675 & 37.325 & 32.625 & 39.700 & 34.885 \\
\hline 23 & 30.850 & 31.550 & 23.600 & 29.625 & 28.717 \\
\hline 24 & 25.350 & 37.000 & 25.200 & 33.300 & 31.040 \\
\hline 25 & 12.725 & 26.650 & 21.275 & 21.825 & 21.695 \\
\hline 26 & 25.150 & 29.450 & 27.150 & 29.100 & 28.037 \\
\hline 29 & 46.300 & 49.025 & 38.900 & 43.325 & 44.245 \\
\hline 31 & 31.125 & 39.400 & 40.400 & 34.800 & 37.212 \\
\hline 32 & 50.200 & 54.550 & 45.075 & 42.125 & 47.900 \\
\hline 33 & 29.875 & 40.675 & 39.000 & 47.175 & 40.420 \\
\hline 34 & 39.325 & 43.800 & 36.350 & 38.725 & 39.682 \\
\hline 35 & 9.050 & 20.050 & 17.475 & 22.250 & 17.827 \\
\hline 36 & 28.350 & 39.800 & 37.800 & 40.875 & 37.792 \\
\hline 37 & 11.775 & 29.100 & 22.700 & 22.575 & 22.800 \\
\hline 38 & 16.000 & 25.875 & 15.225 & 19.950 & 21742 \\
\hline 39 & 29.975 & 45.000 & 34.525 & 41.900 & 38.837 \\
\hline 40 & 33.900 & 53.200 & 40.375 & 40.150 & 43.175 \\
\hline 41 & 30.800 & 33.225 & 29.075 & 29.700 & 30.737 \\
\hline 42 & 44.550 & 43.500 & 30.975 & 49.950 & 41.688 \\
\hline 43 & 40.200 & 32.775 & 23.800 & 41.850 & 34.557 \\
\hline 44 & 44.475 & 43.275 & 37.925 & 36.575 & 40.120 \\
\hline 45 & 26.675 & 33.000 & 32.150 & 32.300 & 31.855 \\
\hline 48 & 34.250 & 41.175 & 35.675 & 39.925 & 37.398 \\
\hline 49 & 25.225 & 39.750 & 36.125 & 50.900 & 39.470 \\
\hline 50 & 36.250 & 39.250 & 33.050 & 49.800 & 39.809 \\
\hline 51 & 14.175 & 10.200 & 8.925 & 14.025 & 11.443 \\
\hline 52 & 31.125 & 37.900 & 32.575 & 40.050 & 35.640 \\
\hline 53 & 45.625 & 52.575 & 39.100 & 52.600 & 47.778 \\
\hline 54 & 42.450 & 51.500 & 36.950 & 54.050 & 46.898 \\
\hline 55 & 27.225 & 37.075 & 35.700 & 42.075 & 36.483 \\
\hline 56 & 17.075 & 32.975 & 29.725 & 38.175 & 30.488 \\
\hline 57 & 24.500 & 33.325 & 45.500 & 50.500 & 40.467 \\
\hline 58 & 30.500 & 44.500 & 31.950 & 50.050 & 40.645 \\
\hline 59 & 40.600 & 42.750 & 37.075 & 38.900 & 39.910 \\
\hline
\end{tabular}


El-Refaey, R.A. et al.

Cont. Table 2

\begin{tabular}{|c|c|c|c|c|c|}
\hline 60 & 36.225 & 36.825 & 32.075 & 38.150 & 35.633 \\
\hline 61 & 21.150 & 47.000 & 40.675 & 51.075 & 42.627 \\
\hline 62 & 13.800 & 24.200 & 18.850 & 21.050 & 20.160 \\
\hline 64 & 40.025 & 47.275 & 41.725 & 54.525 & 46.388 \\
\hline 65 & 18.250 & 27.125 & 26.550 & 18.425 & 23.400 \\
\hline 67 & 43.225 & 36.500 & 34.525 & 40.050 & 37.970 \\
\hline 68 & 33.500 & 35.200 & 36.475 & 44.475 & 37.592 \\
\hline 69 & 35.650 & 36.325 & 38.200 & 46.050 & 39.443 \\
\hline 70 & 40.375 & 40.050 & 46.350 & 36.850 & 38.093 \\
\hline 72 & 15.025 & 35.975 & 35.825 & 42.625 & 34.647 \\
\hline 73 & 12.075 & 14.700 & 21.050 & 23.675 & 18.573 \\
\hline 74 & 5.260 & 25.025 & 20.025 & 22.975 & 19.935 \\
\hline 75 & 38.450 & 47.650 & 41.650 & 55.625 & 46.583 \\
\hline 76 & 42.500 & 46.375 & 42.650 & 52.350 & 46.332 \\
\hline 77 & 30.000 & 45.000 & 38.125 & 52.050 & 37.930 \\
\hline 78 & 29.825 & 38.775 & 36.825 & 48.450 & 39.490 \\
\hline 79 & 23.800 & 40.425 & 39.375 & 53.100 & 41.250 \\
\hline 80 & 39.500 & 38.775 & 36.600 & 54.100 & 42.455 \\
\hline 82 & 22.450 & 38.150 & 38.450 & 52.650 & 39.970 \\
\hline 83 & 23.250 & 36.825 & 34.525 & 35.750 & 33.908 \\
\hline 84 & 26.925 & 44.650 & 39.275 & 60.000 & 45.200 \\
\hline 85 & 26.675 & 37.900 & 38.900 & 50.025 & 39.733 \\
\hline 86 & 33.575 & 36.350 & 37.525 & 49.075 & 39.778 \\
\hline 87 & 27.200 & 44.925 & 43.000 & 16.825 & 37.015 \\
\hline 88 & 27.375 & 32.575 & 35.675 & 44.875 & 30.642 \\
\hline 90 & 39.500 & 39.200 & 40.100 & 49.950 & 42.495 \\
\hline 93 & 20.050 & 33.825 & 30.225 & 29.850 & 29.807 \\
\hline 94 & 43.325 & 47.700 & 46.575 & 61.750 & 50.562 \\
\hline 95 & 19.800 & 33.650 & 29.200 & 25.975 & 28.088 \\
\hline 96 & 33.525 & 39.000 & 36.900 & 52.425 & 41.540 \\
\hline 97 & 18.200 & 32.950 & 36.950 & 51.750 & 37.533 \\
\hline 98 & 33.500 & 40.900 & 39.750 & 52.175 & 42.642 \\
\hline 99 & 28.675 & 32.700 & 18.575 & 30.425 & 27.638 \\
\hline 100 & 34.850 & 44.550 & 41.825 & 58.075 & 46.407 \\
\hline 101 & 42.975 & 34.600 & 35.100 & 56.725 & 41.920 \\
\hline 103 & 34.775 & 36.325 & 37.725 & 47.150 & 39.023 \\
\hline 104 & 38.750 & 38.025 & 36.125 & 45.125 & 39.557 \\
\hline 105 & 35.800 & 38.150 & 35.725 & 40.000 & 37.338 \\
\hline 106 & 16.300 & 34.600 & 36.950 & 43.125 & 34.500 \\
\hline 107 & 10.600 & 31.050 & 26.000 & 28.550 & 25.582 \\
\hline 108 & 53.975 & 42.300 & 37.325 & 41.525 & 42.887 \\
\hline 109 & 45.725 & 47.925 & 40.525 & 48.150 & 46.297 \\
\hline 110 & 33.275 & 42.775 & 42.525 & 53.550 & 43.997 \\
\hline 111 & 4.750 & 12.577 & 10.500 & 22.625 & 13.530 \\
\hline 112 & 10.600 & 31.750 & 29.075 & 27.950 & 26.852 \\
\hline 113 & 23.825 & 29.950 & 33.750 & 38.725 & 32.210 \\
\hline 115 & 10.675 & 25.425 & 21.025 & 38.875 & 25.688 \\
\hline 116 & 39.550 & 41.075 & 37.250 & 52.925 & 43.308 \\
\hline F .test & $* *$ & $* *$ & ** & ** & $\star *$ \\
\hline LSD 0.05 & 4.054 & 4.589 & 2.053 & 5.462 & 3.039 \\
\hline LSD 0.01 & 5.341 & 6.046 & 2.705 & 7.196 & 4.004 \\
\hline
\end{tabular}

** Significant at the 0.01 level of probability. 
Similar results and conclusion were reached by AL-Menoufi \& Hassan, 1977; AL-Menoufi et al. 1985; Lanini \& Kogan, 2005; Abd El-Hamid \& El-Khanagry, 2006; Goldwasser et al., 2001). These results may indicate that both genotypes no 51 and no. 111 had the least R \% for most of the cuts and seasonal yield. Genotype no. 111 is commercial cultivar, Helali. Helali cv. has high potential and high-yielding ability in comparison with other cultivars. It was bred for relatively high ability to rapid re-growth which may be associated with good tolerance to biotic and abiotic stresses. Therefore, to be less affected due to dodder infestation it could be expected in comparison with other material under evaluation. Moreover, as it was reported by (Abd ElHamid \& El-Khanagry, 2006), about $85 \%$ of farmers' fields are infested with dodder. Existing of genotype no.51 among farmers' seed lots which retains some tolerance to dodder infestation is, also, expected due to co-evolution among parasite-host relationships.

$2^{\text {nd }}$ season $(2009 / 10)$ :

The mean squares of fresh as well as dry forage yield reduction percentages at different cuts and seasonal yield in the $2^{\text {nd }}$ season $(2009 / 10)$ are presented (Table 3 ).

Table 3 : Mean squares of fresh and dry forage yields reduction percent of Egyptian clover-genotypes infested by dodder at different cuts and seasonal yield in $2^{\text {nd }}$ season (2009/2010).

\begin{tabular}{|c|c|c|c|c|c|c|}
\hline \multirow[b]{2}{*}{ S.O.V. } & \multirow[b]{2}{*}{$\begin{array}{l}\text { d. } \\
\text { f. }\end{array}$} & \multicolumn{5}{|c|}{ Mean squares of reduction of fresh forage yield (FFY R \%) } \\
\hline & & $1^{\text {st }}$ Cut & $2^{\text {nd }}$ Cut & $3^{\text {rd }} \mathrm{Cu} 3$ & $4^{\text {th }}$ Cut & $\begin{array}{c}\text { Seasonal } \\
\text { vield }\end{array}$ \\
\hline Genotypes & 26 & $216.47^{* *}$ & $200.41^{* *}$ & $581.65^{* *}$ & $650.92^{*}$ & $269.5^{* 1}$ \\
\hline Error & 78 & 2.88 & 2.23 & 2.03 & 3.50 & 0.776 \\
\hline & & \multicolumn{5}{|c|}{ Mean squares of reduction of dry forage yield (DFY R \%) } \\
\hline Genotypes & 26 & 277.81 & 129.88 & $608.31^{\prime \prime}$ & 720.17 & 365.91 \\
\hline Error & 78 & 11.92 & 12.99 & 17.73 & 15.88 & 4.47 \\
\hline
\end{tabular}

**: Significant at the 0.01 levels of probability.

Highly significant differences are shown (Table 3) among evaluated genotypes at all cuts and the seasonal yield for $\mathrm{R} \%$ due to dodder infestation of both fresh and dry forage yields in $2^{\text {nd }}$ season (2009/10). The means of FFY R\% as well as DFY R\% of 27 genotypes at different cuts and seasonal yield in $2^{\text {nd }}$ season $(2009 / 10)$ are presented (Table 4). It is shown from the recorded data that genotype no.29 had the highest FFY R\% (44.0\%) due to dodder infestation for the seasonal yield in $2^{\text {nd }}$ season $(2009 / 10)$. On the other hand, genotype no.111 had the least R\% (10.1\%) followed by genotype no. 51 where $\mathrm{R} \%=10.3$ for the same trait and it is shown to retain good tolerance to dodder infestation.

It is shown from the results that genotype no.29 had the highest DFY $\mathrm{R} \%$ (50.90\%). While genotype no.111 had the least $\mathrm{R} \%$ (14.5\%) and it may be considered most tolerant genotype under the dodder infestation among the tested genotypes. Likewise, genotype no.111 was followed by genotype no. 51 which had R\% $=14.6 \%$ of DFY. In general, the berseem genotypes under investigation manifested highly significant differences at all cuts and seasonal dry yield for R\% due to dodder infestation. These results are in 
agreement with similar investigations by AL-Menoufi \& Hassan, 1977; ALMenoufi et al. 1985; Lanini \& Kogan, 2005; Abd El-Hamid \& El-Khanagry, 2006; Goldwasser et al., 2001). Similarly, the DFY R \% by dodder infestation was existed. Its grand mean increased from the $1^{\text {st }}$ cut to $2^{\text {nd }}$ cut then decreased in the $3^{\text {rd }}$ cut and start to increase again in the $4^{\text {th }}$ cut starting with $R \%=20.1 \%, 41.5 \%, 29.8 \%$, and $31.1 \%$, respectively, while, $R \%$ for seasonal yield are $32.4 \%$.

Table 4 : Means of fresh and dry forage yield of Egyptian clovergenotypes infested by dodder at different cuts and seasonal yield in $2^{\text {nd }}$ season $(2009 / 10)$.

\begin{tabular}{|c|c|c|c|c|c|c|c|c|c|c|}
\hline \multirow{2}{*}{ Genotypes } & \multicolumn{2}{|c|}{$1^{\text {st }}$ Cut } & \multicolumn{2}{|c|}{$2^{\text {nd }}$ Cut } & \multicolumn{2}{|c|}{$3^{\text {rd }} \mathrm{Cu} 3$} & \multicolumn{2}{|c|}{$4^{\text {th }}$ Cut } & \multicolumn{2}{|c|}{ Seasonal yield } \\
\hline & FFY & $\overline{\text { DFY }}$ & FFY & DFY & FFY & $\overline{D F Y}$ & FFY & DFY & FFY & DFY \\
\hline 2 & 29.70 & 39.55 & 20.20 & 44.35 & 34.20 & 28.90 & 32.50 & 30.22 & 29.50 & 37.00 \\
\hline 8 & 7.60 & 14.82 & 21.90 & 44.45 & 37.80 & 37.47 & 42.10 & 44.15 & 29.90 & 39.10 \\
\hline 12 & 7.20 & 11.75 & 7.80 & 33.40 & 18.60 & 27.22 & 22.50 & 23.72 & 15.10 & 28.02 \\
\hline 14 & 14.40 & 24.80 & 25.60 & 47.10 & 17.60 & 23.07 & 21.90 & 24.75 & 20.10 & 28.94 \\
\hline 15 & 8.10 & 14.35 & 10.30 & 36.32 & 25.40 & 23.65 & 27.90 & 28.55 & 19.20 & 28.41 \\
\hline 16 & 14.90 & 18.12 & 23.10 & 47.42 & 19.70 & 23.55 & 34.00 & 37.87 & 23.80 & 30.66 \\
\hline 21 & 2.10 & 21.90 & 20.80 & 42.24 & 36.10 & 36.85 & 26.70 & 26.42 & 25.30 & 34.66 \\
\hline 23 & 16.10 & 23.70 & 21.50 & 45.15 & 41.90 & 43.18 & 38.90 & 36.87 & 31.40 & 38.66 \\
\hline 25 & 3.30 & 19.40 & 21.80 & 43.37 & 31.80 & 38.25 & 35.50 & 38.62 & 26.40 & 36.02 \\
\hline 26 & 25.60 & 32.47 & 24.40 & 46.45 & 29.00 & 26.57 & 43.10 & 48.37 & 30.90 & 39.49 \\
\hline 29 & 23.60 & 29.00 & 34.80 & 52.70 & 55.90 & 50.95 & 51.90 & 51.87 & 44.00 & 50.92 \\
\hline 35 & 7.70 & 8.82 & 10.00 & 33.82 & 15.80 & 27.02 & 24.20 & 23.20 & 15.10 & 24.24 \\
\hline 37 & 8.60 & 16.35 & 16.80 & 40.67 & 20.50 & 21.12 & 23.60 & 22.05 & 18.20 & 25.19 \\
\hline 38 & 6.10 & 16.57 & 12.60 & 36.35 & 26.80 & 26.42 & 27.50 & 28.72 & 20.10 & 25.70 \\
\hline 51 & 3.30 & 3.25 & 12.40 & 33.20 & 17.90 & 12.17 & 5.20 & 5.50 & 10.30 & 14.61 \\
\hline 56 & 25.90 & 30.50 & 21.90 & 42.87 & 35.40 & 16.22 & 46.80 & 47.47 & 33.60 & 39.81 \\
\hline 62 & 5.00 & 10.62 & 11.60 & 32.85 & 30.80 & 44.50 & 22.60 & 21.27 & 19.10 & 31.59 \\
\hline 65 & 9.40 & 18.17 & 8.70 & 35.87 & 19.20 & 13.50 & 26.90 & 24.95 & 17.20 & 30.21 \\
\hline 73 & 15.30 & 17.02 & 16.90 & 40.40 & 19.20 & 17.10 & 11.40 & 9.17 & 15.80 & 17.77 \\
\hline 74 & 8.00 & 17.80 & 14.20 & 40.45 & 25.40 & 37.80 & 22.20 & 24.95 & 18.30 & 31.17 \\
\hline 93 & 1.60 & 27.80 & 32.90 & 53.25 & 62.20 & 62.62 & 65.60 & 65.82 & 36.40 & 56.41 \\
\hline 95 & 18.20 & 24.35 & 22.70 & 43.20 & 35.30 & 35.42 & 31.40 & 32.72 & 27.90 & 35.53 \\
\hline 99 & 19.10 & 28.80 & 18.50 & 42.05 & 33.10 & 32.77 & 28.90 & 33.12 & 26.10 & 35.87 \\
\hline 107 & 17.90 & 23.52 & 22.10 & 44.02 & 39.80 & 41.37 & 43.60 & 44.62 & 32.40 & 42.28 \\
\hline 111 & 3.00 & 7.47 & 8.50 & 33.25 & 10.80 & 10.75 & 15.50 & 12.25 & 10.10 & 14.54 \\
\hline 112 & 10.10 & 17.97 & 16.00 & 39.62 & 18.40 & 24.65 & 22.70 & 24.47 & 17.70 & 26.30 \\
\hline 115 & 18.90 & 25.80 & 25.10 & 45.57 & 22.10 & 21.42 & 27.90 & 30.52 & 23.70 & 30.82 \\
\hline F. test & ** & ** & ** & ** & ** & ** & ** & ** & ** & ** \\
\hline LSD 0.05 & 2.39 & 4.86 & 2.11 & 5.07 & 2.01 & 5.93 & 2.64 & 5.61 & 29.50 & 37.00 \\
\hline LSD 0.01 & 3.17 & 6.45 & 2.79 & 6.73 & 2.66 & 7.86 & 3.49 & 7.44 & 29.90 & 39.10 \\
\hline
\end{tabular}

${ }^{*}$ : Significant at the 0.01 levels of probability.

Mean squares of $\mathrm{R} \%$ of total chlorophyll content due to dodder infestation at different cuts and their mean in $2^{\text {nd }}$ season $(2009 / 010)$ and $3^{\text {rd }}$ season (2010/2011) are presented (Table 5). It could be shown from the results that highly significant differences existed among the evaluated genotypes at all cuts and their mean for R \% of total chlorophyll content in $2^{\text {nd }}$ season $(2009 / 10)$ and $3^{\text {rd }}$ season $(2010 / 2011)$. 
Table 5 : Mean squares of total chlorophyll reduction percent $(R \%)$ of Egyptian clover-genotypes infested by dodder at different cuts and their means in $2^{\text {nd }}$ season $(2009 / 10)$ and $3^{\text {rd }}$ season (2010/2011).

\begin{tabular}{|c|c|c|c|c|c|c|}
\hline \multirow{2}{*}{ S. O. V. } & \multirow{2}{*}{ d. f. } & $1^{\text {st }}$ Cut & $2^{\text {nd }}$ Cut & $3^{\text {rd }}$ Cut & $4^{\text {th }}$ Cut & Mean \\
\hline & & \multicolumn{5}{|c|}{$2^{\text {nd }}$ season $(2009 / 10)$} \\
\hline Replications & 3 & 0.539 & 2.650 & 1.139 & 0.593 & 0.793 \\
\hline Genotypes & 26 & 65.80 & 69.56 & 111.56 & 82.29 & 65.12 \\
\hline \multirow[t]{2}{*}{ Error } & 78 & 4.91 & 6.22 & 5.07 & 6.99 & 1.43 \\
\hline & & \multicolumn{5}{|c|}{$3^{\text {rd }}$ season $(2010 / 2011)$} \\
\hline Replications & 3 & 0.548 & 2.340 & 1.138 & 0.459 & 0.835 \\
\hline Genotypes & 26 & 13.07 & 15.29 & 27.62 & 21.88 & $9.77^{\prime \prime}$ \\
\hline Error & 78 & 1.731 & 1.631 & 1.009 & 1.553 & 0.164 \\
\hline
\end{tabular}

Mean of R \% for total chlorophyll content of 27 genotypes at different cuts and their mean in $2^{\text {nd }}$ season (2009/010) are presented (Table 6).

Table 6: Mean of total chlorophyll $\mathbf{R} \%$ at different cuts and seasonal yield of clover in $2^{\text {nd }}$ season $(2009 / 010)$.

\begin{tabular}{|c|c|c|c|c|c|}
\hline $\mathbf{G}$ & Cut1 & Cut2 & Cut3 & Cut4 & mean \\
\hline 2 & 12.900 & 12.875 & 14.975 & 14.250 & \\
\hline 8 & 19.650 & 13.325 & 12.425 & 8.425 & 13.4 \\
\hline 12 & 9.675 & 7.450 & 12.800 & 9.425 & 13.6 \\
\hline 14 & 11.605 & 11.450 & 9.125 & 12.775 & 9.9 \\
\hline 15 & 7.425 & 6.175 & 10.925 & 11.550 & 11.3 \\
\hline 16 & 12.325 & 10.100 & 11.800 & 15.650 & 9.1 \\
\hline 21 & 13.900 & 17.00 & 16.350 & 14.700 & 12.6 \\
\hline 23 & 10.200 & 14.500 & 10.725 & 13.575 & 15.6 \\
\hline 25 & 10.900 & 12.700 & 12.750 & 15.725 & 12.3 \\
\hline 26 & 10375 & 15.00 & 16.550 & 20.125 & 13.1 \\
\hline 29 & 15.750 & 18.700 & 29.200 & 25.00 & 15.7 \\
\hline 35 & 6.550 & 6.875 & 8.750 & 9.575 & 22.3 \\
\hline 37 & 6.625 & 8.900 & 8.925 & 10.100 & 7.9 \\
\hline 38 & 8.200 & 7.750 & 9.775 & 9.500 & 8.7 \\
\hline 51 & 6.575 & 8.900 & 8.075 & 10.800 & 8.9 \\
\hline 56 & 9.200 & 7.275 & 10.275 & 14.100 & 8.6 \\
\hline 62 & 6.825 & 8.550 & 10.050 & 9.125 & 10.3 \\
\hline 65 & 8.650 & 10.875 & 14.625 & 12.950 & 8.7 \\
\hline 73 & 8.675 & 7.425 & 10.925 & 11.125 & 11.8 \\
\hline 74 & 6.375 & 8.650 & 10.125 & 9.875 & 9.6 \\
\hline 93 & 19.650 & 22.750 & 25.900 & 20.800 & 8.9 \\
\hline 95 & 13.875 & 17.175 & 18.575 & 20.350 & 22.1 \\
\hline 99 & 19.425 & 13.675 & 15.250 & 16.325 & 17.5 \\
\hline 107 & 14.800 & 14.600 & 18.950 & 15.625 & 16.1 \\
\hline 111 & 9.325 & 7.028 & 6.650 & 5.925 & 16.0 \\
\hline 112 & 8.700 & 11.575 & 7.450 & 9.825 & 7.2 \\
\hline 115 & 8.850 & 13.350 & 13.200 & 7.900 & 9.5 \\
\hline F. test & ** & * * & $* *$ & $* *$ & 11.0 \\
\hline $\begin{array}{c}\text { L.S.D0.05 } \\
0.01\end{array}$ & $\begin{array}{c}3.12 \\
4.138\end{array}$ & $\begin{array}{c}3.511 \\
4.6570\end{array}$ & $\begin{array}{c}3.17 \\
4.205\end{array}$ & $\begin{array}{l}3.723 \\
4.938\end{array}$ & $* *$ \\
\hline
\end{tabular}

** Significant at the 0.01 level of probability, respectively. 
Significant differences among genotypes at all cuts concerning total chlorophyll R \% was observed. Genotype no. 29 had the highest R\% (22.3\%) of total chlorophyll by dodder infestation while genotype no.111 had the lowest R \% (7.2\%) and it was followed by the genotype no.35 and 51, where they had $7.9 \%$ and $8.7 \%$ of reduction for the total chlorophyll content. The obtained results for total chlorophyll $\mathrm{R} \%$ are in agreement with those obtained by Dinelli et al., 1993; Parker and Riches, 1993; Soliman, 2002; and Soliman \& Abd El-Hamid, 2009.

$3^{\text {rd }}$ Season (2010/11):

Mean squares of fresh and dry forage yield $\mathrm{R} \%$ due to dodder infestation in $3^{\text {rd }}$ season (2010/11) are presented (Table 7). Highly significant differences among evaluated genotypes at all cuts and seasonal yield for fresh as well as dry forage yield $\mathrm{R} \%$ due to dodder infestation in $3^{\text {rd }}$ season $(2010 / 11)$ are detected.

Table 7 : Mean squares of fresh and dry forage yield reduction (R \%) of berseem genotypes infested by dodder at different cuts and seasonal yield in $3^{\text {rd }}$ season (2010/11).

\begin{tabular}{|c|c|c|c|c|c|c|}
\hline \multirow{2}{*}{ S. O.V. } & \multirow{2}{*}{ d. f. } & \multicolumn{5}{|c|}{ Reduction of Fresh Forage Yield (R \%) } \\
\hline & & $1^{\text {st }}$ Cut & $2^{\text {nd }}$ Cut & $3^{\text {rd }}$ Cut & $4^{\text {th }}$ Cut & Seasonal yield \\
\hline Replication & 3 & 8.373 & 10.611 & 1.824 & 46.285 & 7.710 \\
\hline Genotypes & 13 & $345.61^{* *}$ & $301.32^{* *}$ & $294.76^{* *}$ & $547.40^{* *}$ & $327.47^{*}$ \\
\hline Error & 39 & 3.67 & 6.54 & 4.54 & 8.87 & 1.86 \\
\hline & & \multicolumn{5}{|c|}{ Reduction of Dry Forage Yield (R \%) } \\
\hline Replication & 3 & 17.70 & 23.68 & 5.70 & 53.42 & 7.59 \\
\hline Genotypes & 13 & $327.81^{* *}$ & $332.21^{*}$ & $317.85^{* *}$ & $573.62^{* *}$ & 355.90 \\
\hline Error & 39 & 5.32 & 7.11 & 5.62 & 8.87 & 2.40 \\
\hline
\end{tabular}

${ }^{\star \star}$ : significant at the 0.01 levels of probability.

Mean of FFY R \% at different cuts and seasonal yield due to dodder infestation in $3^{\text {rd }}$ season $(2010 / 11)$ are shown (Table 8$)$. It is shown from the results that genotype (Giza 6) had the highest R\% (43.3\%) of the seasonal yield. On the other hand, the lowest R\% (11.1\%) recorded for genotype no.1110 (parent) then followed by genotype no. 111 (progeny) where it R $\%=$ $11.3 \%$ for $F F Y$ in $3^{\text {rd }}$ season $(2010 / 11)$. Similarly, mean of $R \%$ for dry forage yield due to dodder infestation at different cuts and their seasonal yield in $3^{\text {rd }}$ season are presented in Table (8). It could be indicated from the data that genotype no. 51 had the lowest R\% (11.9\%) among the 14 genotypes under evaluation then followed by genotype no.111 where its $\mathrm{R} \%=12.4 \%$. However, the highest R \% (46.5\%) was obtained by genotype Giza-6. Highly significant differences of $\mathrm{R} \%$ among evaluated genotypes at all cuts and the seasonal yield in $3^{\text {rd }}$ season $(2010 / 11)$ were detected. 
Table 8 : Mean of fresh (F) and dry (D) forage yields reduction (FY R \%) of berseem genotypes infested by dodder at different cuts and seasonal yield in $3^{\text {rd }}$ season $(2010 / 11)$.

\begin{tabular}{|c|c|c|c|c|c|c|c|c|c|c|}
\hline \multirow{2}{*}{ Genotypes } & \multicolumn{2}{|c|}{$1^{\text {st }}$ Cut } & \multicolumn{2}{|c|}{$2^{\text {nd }}$ Cut } & \multicolumn{2}{|c|}{$3^{\text {rd }} \mathrm{Cu} 3$} & \multicolumn{2}{|c|}{$4^{\text {th }}$ Cut } & \multicolumn{2}{|c|}{ Seasonal yield } \\
\hline & FFY & DFY & FFY & DFY & FFY & DFY & FFY & DFY & FFY & DFY \\
\hline 120 & 14.3 & 18.8 & 15.90 & 17.5 & 10.7 & 23.5 & 18.00 & 28.7 & 14.9 & 23.0 \\
\hline 350 & 6.9 & 12.4 & 16.10 & 16.2 & 14.5 & 21.2 & 19.2 & 24.9 & 15.2 & 20.1 \\
\hline 510 & 7.1 & 12.9 & 15.20 & 16.6 & 9.8 & 13.7 & 13.5 & 22.5 & 11.8 & 17.3 \\
\hline 730 & 10.3 & 12.4 & 8.90 & 9.4 & 10.8 & 11.2 & 31.3 & 37.1 & 16.1 & 19.9 \\
\hline 740 & 9.3 & 11.3 & 14.50 & 17.3 & 18.4 & 21.9 & 15.8 & 22.5 & 15.0 & 19.6 \\
\hline 1110 & 6.1 & 5.2 & 9.20 & 11.6 & 12.0 & 12.7 & 13.8 & 25.1 & 11.1 & 15.3 \\
\hline 12 & 13.4 & 14.7 & 17.10 & 17.9 & 11.4 & 14.6 & 19.0 & 21.2 & 15.5 & 17.7 \\
\hline 35 & 6.5 & 10.8 & 16.50 & 17.7 & 14.9 & 15.5 & 19.6 & 18.0 & 15.7 & 16.4 \\
\hline 51 & 6.6 & 12.5 & 14.10 & 14.7 & 9.7 & 10.5 & 12.8 & 10.9 & 11.9 & 11.9 \\
\hline 73 & 10.1 & 13.2 & 10.40 & 12.3 & 11.4 & 9.9 & 29.5 & 29.2 & 16.0 & 17.2 \\
\hline 74 & 8.8 & 10.6 & 14.80 & 14.2 & 17.4 & 17.1 & 15.0 & 14.8 & 14.5 & 14.8 \\
\hline 111 & 5.1 & 10.2 & 11.00 & 10.2 & 11.8 & 11.9 & 14.5 & 15.1 & 11.3 & 12.4 \\
\hline Gemmiza1 & 23.8 & 27.7 & 29.90 & 32.1 & 31.8 & 33.1 & 38.7 & 42.1 & 32.2 & 35.6 \\
\hline Giza 6 & 39.5 & 41.3 & 41.10 & 43.3 & 38.1 & 39.9 & 52.9 & 55.7 & 43.3 & 46.5 \\
\hline F .test & ** & ** & ** & ** & ** & ** & ** & ** & $*$ & ** \\
\hline L.S. D 0.5 & 2.74 & 3.30 & 3.76 & 3.81 & 3.05 & 3.39 & 4.26 & 4.60 & & 2.23 \\
\hline L.S. D 0.1 & 3.67 & 4.42 & 4.90 & 5.11 & 4.08 & 4.54 & 5.71 & 5.71 & & 2.97 \\
\hline
\end{tabular}

**: significant at the 0.01 levels of probability.

Mean of total chlorophyll reduction ( $\mathrm{R} \%$ ) due to dodder infestation at different cuts and their mean in $3^{\text {rd }}$ season $(2010 / 11)$ are presented in Table (9).

Table 9: Mean of total chlorophyll reduction ( $R \%)$ of berseem genotypes infested by dodder at different cuts and their means in $3^{\text {rd }}$ season $(2010 / 11)$.

\begin{tabular}{|c|c|c|c|c|c|}
\hline Genotypes & $\mathbf{1}^{\text {st }}$ Cut & $\mathbf{2}^{\text {nd }}$ Cut & $\mathbf{3}^{\text {rd }}$ Cu3 & $\mathbf{4}^{\text {th }}$ Cut & Mean \\
\hline $120^{p}$ & 7.8 & 6.4 & 7.2 & 6.4 & 6.9 \\
\hline $350^{p}$ & 9.2 & 8.7 & 9.9 & 7.5 & 8.8 \\
\hline $510^{p}$ & 5.9 & 5.7 & 5.8 & 4.4 & 5.5 \\
\hline $730^{p}$ & 6.9 & 4.8 & 7.6 & 2.3 & 5.5 \\
\hline $740^{p}$ & 8.7 & 7.9 & 10.2 & 6.1 & 8.2 \\
\hline $1110^{p}$ & 4.5 & 8.6 & 2.5 & 4.9 & 5.2 \\
\hline $12^{g}$ & 7.2 & 12.8 & 5.3 & 7.1 & 8.2 \\
\hline $35^{9}$ & 8.0 & 6.2 & 6.6 & 7.3 & 7.1 \\
\hline $51^{g}$ & 7.6 & 6.3 & 8.1 & 3.8 & 6.5 \\
\hline $73^{g}$ & 8.4 & 7.8 & 9.9 & 5.4 & 8.0 \\
\hline $74^{g}$ & 4.9 & 7.2 & 6.0 & 5.2 & 5.8 \\
\hline $111^{g}$ & 4.4 & 6.9 & 1.2 & 6.6 & 4.8 \\
\hline Gemmiza1 & 9.4 & 9.3 & 7.4 & 10.6 & 9.1 \\
\hline Giza 6 & 9.9 & 8.1 & 8.1 & 10.7 & 9.4 \\
\hline F .test & $\star \star$ & $\star \star$ & & & \\
\hline L.S. D 0.5 & 1.882 & 1.827 & 1.437 & 1.282 & 0.579 \\
\hline L.S. D 0.1 & 2.519 & 2.445 & 1.923 & 2.386 & 0.775 \\
\hline
\end{tabular}

${ }^{* *}$ : significant at the 0.01 levels of probability. P: parent, g: progeny, c: check. 
Highly significant differences among all the genotypes under evaluation at all the cuts and their mean are manifested. It is clear that Giza 6 had the highest (9.4) total chlorophyll R \%, while, genotype no.111 had the least (4.8) total chlorophyll R \% then followed by genotype no.1110 and genotype no.510 where $\mathrm{R} \%$, values were 5.2 and 5.5 , respectively. The obtained results are in line with the following research findings by AL-Menoufi et al. 1985; Lanini \& Kogan, 2005; Abd El-Hamid \& El-Khanagry, 2006; Goldwasser et al., 2001.

\section{CONCULUSION}

Even though resistance/tolerance among and within crop plants to pests, especially for parasitic weed like dodder is difficult to obtain, highly significant differences among the evaluated berseem genotypes for fresh and dry forage yield reduction percentages due to

dodder infestation in all cuts and seasonal yield during the study were detected

These differences are highly supported with obtaining less reduction percentages of fresh as well as dry forage yields for the tolerant berseem genotypes due to infestation with dodder. Indictor's traits which are highly associated i.e. total chlorophyll R\%, CP R\%, CF R\%, and ash well performed for tolerant genotypes in comparison with the sensitive ones. The obtained results may be encouraging to use the tolerant genotypes, which had the least reduction percentages due to dodder infestation, for developing compost or synthetic cultivar(s) which retain tolerance to dodder infestation. This cultivar could be used by itself as a way of control of dodder in farmers' fields or integrated with the other factors of dodder control.

\section{REFERENCES}

Abd El-Hady, A. (1993). Potassium and its effects on crop productivity in Egyptian soils. Bull. (In Arabic). Soils and Water Res. Inst., Agric. Res. Center, Giza, Egypt.

Abd El-Hamid, M.M. and S.S. El-Khanagry (2006). Studies on dodder Cuscuta spp. infestation in clover T. alexandrinum L. fields in some governorates in Nile Delta. Egypt. J. Agric. Res., 84 (1), 2006.

Abd El-Wahed, R. (1996). Studies on the effect of certain herbicides of dodder C. spp. control in certain leguminous crops. Ph. D. Thesis, Fac. Agric. Cairo Univ.

ACSAD, 2008. Development Program for Feed Sources in Arab Countries; Feed Budget in Egypt, Arab Republic of, Damascus 2008. (In Arabic).

Al-Menoufi, O.A. and M. Hassan. (1977). Studies on the parasitism of Cuscuta spp. Series 2. The effect of dodder Cuscuta planiflora Ten. on the production and chemical composition of Egyptian clover Trifolium alexandrinum L. Alex. J. Agric. Res. 25 (2):337-341.

Al-Menoufi O.A., M. A. Rezk, A.K.Mostafa and S. EL-Shair (1985). Studies on the parasitism of Cuscuta spp. 6- Losses in Egyptian clover and flax due to dodder infection. J. Agric. Sci. Mansoura Univ. 10(4):12621266. 
Comstock, R.E., and H.F. Robinson. (1952). Genetic parameters, their estimation and significance. Proc. $6^{\text {th }}$ Int. grasslands Congr. 1; 284291.

Dinelli, C., A. Bone Hi and E. Tibiletti. (1993). Play to synthetic and accessory pigments in Cuscuta campestris Yuncker and some host species. Weed Res.33-253-260.

El-Nahrawy, M.A. (2009a). 'Berseem Clover and Its Vital Role in Egyptian Agriculture.' FAO Regional Expert Consultation Meeting on Irrigated Fodder Crops in the Near East Region-Forage Production, Conservation, and Utilization for Sustainable Animal Production. IAV Hassan II, 15-19 June 2009, Rabat, Morocco.

El-Nahrawy, M.A. (2009b). Forage crops status in Egypt.' FAO Regional Expert Consultation Meeting on Irrigated Fodder Crops in the Near East Region-Forage Production, Conservation, and Utilization for Sustainable Animal Production. IAV Hassan II, 15-19 June 2009, Rabat, Morocco.

Goldwasser. Y., R.L.Worobel and W.T. Lanini (2001). Tolerance of tomato varieties to lespedeza dodder. Weed Sci.49:520 - 523.

Graves, W. L., W. A. Williams and C. D. Thomsen (1996). Berseem clover: A winter annual forage for California agriculture. University of California Division of Agric. and Natural Resources, Publication No. 21536, pp: 12.

Kennedy, P.B. and W.W. Mackie (1925). Berseem or Egyptian clover Trifolium alexandrinum L.. A preliminary Report. Bull. 389 June, 1925 Univ. of Calf. Printing Office, Berkeley, Calf.

Lanini, W. T. and M. Kogan (2005). Biology and management of Cuscuta in crops. Ciencia E Inestigacion Agraria.Vol. 32 N 3 Sept.-Dec. 2005. Pp. 165-179.

Parker, C. and C.R. Riches.(1993). Parasitic weeds of the world: Biology and control, CAB International, Wallingford, UK.

Snedecor, G. W. and W. G. Cochran (1971). Statistical Methods $6^{\text {th }}$ ed., lowa State Univ., Ames, lowa, U.S.A.

Soliman, I.E. (2002). Herbicidal activity evaluation of some herbicides used to control of dodder Cuscuta spp. in some field crops. Ph.D. Thesis, Fac. of Agric. Mansoura Univ.

Soliman, I.E. and M.M. Abd El-hamid (2009). Effect of sowing methods and some weed control treatments on dodder control in clover crop. J. Agric. Sci. Mansoura Univ. 34(4):3211-3221.

Topps, T. H. and R. L. Wain (1957). Investigation on fungicides III. The fungitoxicity of 3- and 5 alkyl salicylanilide and P-chloronilines. Ann. Applied Biol., 45: 506-514. 
التحمل النسبى لبعض التراكيب الوراثية للبرسيم المصرى للاصابة بالحامول

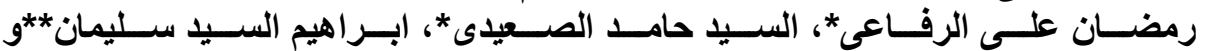

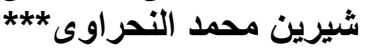

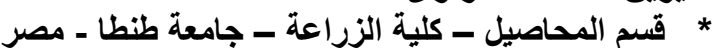

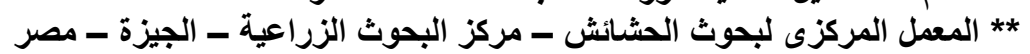

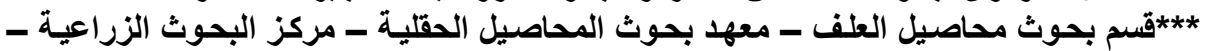

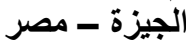

ثلاث تجارب حقلية أجريت بمزرعة محطة البحوث الزراعية بسخا ـ كفر الثيخ خلال ثلاث مواسم

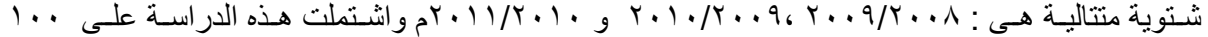

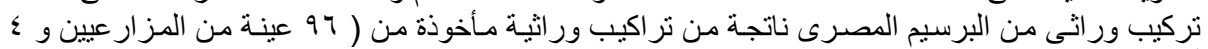

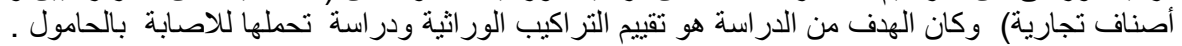

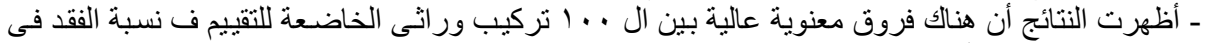

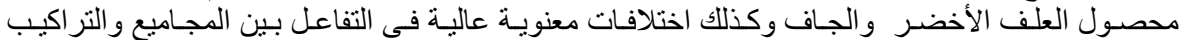

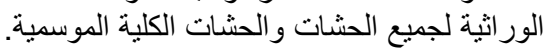

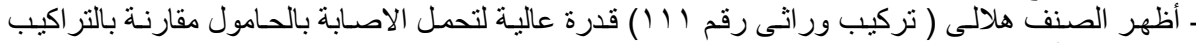

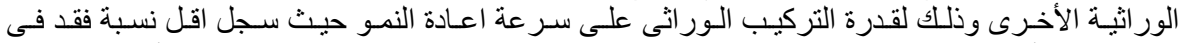

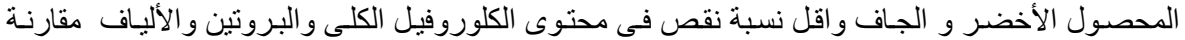
بالتر اكيب الور اثية الأخرى التى تم اصابتها بالحامول.

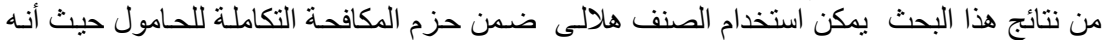
تحمل الاصابة بالحامول مقارنة بغيره من التر اكيب الور اثية الأخرى محل الدراسة. 